\title{
Albert Memmi : Portrait du colonisé, précédé d'un Portrait du colonisateur
}

Note sur une postface autographe inédite

\section{Guy Dugas}

\section{(2) OpenEdition}

\section{Journals}

Édition électronique

URL : http://journals.openedition.org/genesis/621

DOI : 10.4000/genesis.621

ISSN : 2268-1590

Éditeur :

Presses universitaires de Paris Sorbonne (PUPS), Société internationale de génétique artistique littéraire et scientifique (SIGALES)

\section{Édition imprimée}

Date de publication : 30 octobre 2011

Pagination : 119-126

ISBN : 978-2-84050-804-5

ISSN : 1167-5101

\section{Référence électronique}

Guy Dugas, «Albert Memmi : Portrait du colonisé, précédé d'un Portrait du colonisateur », Genesis [En ligne], 33 | 2011, mis en ligne le 23 octobre 2013, consulté le 30 avril 2019. URL : http:// journals.openedition.org/genesis/621; DOI : 10.4000/genesis.621 


\section{Albert Memmi : Portrait du colonisé, précédé d'un Portrait du colonisateur Note sur une postface autographe inédite}

Guy Dugas

$\mathrm{I}$ l a souvent été souligné - et par Albert Memmi lui-même - combien La Statue de sel (Corréa, 1953), son premier roman, puis Agar (Corréa, 1955) qui en est le prolongement autobiographique, constituent la matrice de toute son œuvre ultérieure, ouvrages de fiction et essais confondus. Des essais qui prennent d'abord la forme de portraits - un genre littéraire qui eut son heure de gloire aux XVII ${ }^{e}$ et $X I_{I I}{ }^{e}$ siècles avec les moralistes, mais actuellement fort peu utilisé.

Réinstallé en Tunisie à la fin de ses études supérieures, Memmi enseigne, au moment où il a l'idée de ces portraits, au lycée Carnot, le lycée français de Tunis, avant d'être nommé directeur d'un centre de psychopédagogie. Il vit alors du dedans la décolonisation au Maghreb, en intellectuel militant, mais avec cette différence majeure qu'il n'est pas un intellectuel ou un militant comme les autres, parce qu'il est un juif arabe - ce dont il ne tardera pas à se rendre compte et qui justifiera au printemps 1956 son nouveau et définitif départ en France.

Pour comprendre les difficultés de l'itinéraire du colonisé acculturé, celles du « colonisateur de bonne volonté », « il me fallait, dit Memmi, comprendre le colonisateur et le colonisé, et peut-être toute la relation et la situation coloniales ». C'est ainsi qu'il est amené à faire une série de conférences ou de chroniques dans les journaux locaux sur la psychologie de l'un et de l'autre, et sur l'analyse de la relation qui les unit en les opposant. Le premier portrait à avoir vu le jour dans l'esprit de l'auteur, et à partir de l'analyse de sa condition même, est celui du colonisé lequel, après quelques touches et retouches ${ }^{1}$, paraîtra dans Esprit en mai 1957. Pourtant, avant même que celui-ci ne soit publié, Memmi s'est déjà rendu compte que chaque trait, chaque détail qu'il dépeint de cette figure de l'opprimé ne donne «qu'un aspect partiel de ce qu'[il] voudrai[t] dire, qui [le] laisse insatisfait, pressé de dire la suite, sans laquelle [s]a pensée est mutilée, presque fausse 2 ». Il se met donc presque simultanément à la rédaction d'un " Portrait du colonisateur de bonne volonté » (Les Temps modernes, avril 1957), venant prolonger, corriger, compléter le premier.
Les deux essais (le « Portrait du colonisé » restant tel quel, celui du colonisateur étant légèrement augmenté), seront ensuite très rapidement réunis par Corréa, éditeur de ses deux romans antérieurs, pour paraître en volume dès juin 19573 .

L'étude des manuscrits de ces deux textes, conservés par l'auteur, fait apparaître nombre de modifications significatives que seule une édition génétique complète permettra d'analyser en profondeur ${ }^{4}$. On y suit parfaitement la réflexion de l'auteur, qui brosse, touche après touche, une série de portraits du colonisé comme du colonisateur, selon un effet de miroir d'une catégorie à l'autre : au colonisateur qui se refuse correspond un colonisé honteux, désireux de disparaître dans l'assimilation ; le colonisateur qui s'accepte (le colonialiste) appelle le colonisé qui se revendique et le montre dans sa révolte.

Cet ensemble de portraits s'inscrivant au sein d'une seule et même dynamique, « celle de la relation coloniale, qui transforme le colonial en C[olonisateu $]^{\mathrm{r}}$ ou en C[olonial] iste, et fait souhaiter l'assimilation au C[olonis]é, puis le pousse à la révolte 5 ».

Le plan du volume est nettement organisé en deux parties (colonisateur/colonisé) séparées dès le titre, qui marque la prévalence accordée au Portrait du colonisé6. Chaque portrait [lire la suite de la présentation p. 126]

1. Voir notamment « La psychologie du colonisé », conférence du 9 mars 1956 au lycée Carnot.

2. Journal intime, dimanche 8 avril [1956].

3. On pense généralement que c'est cette édition originale qui bénéficia de la célèbre préface de Sartre, mais il n'en fut rien. Cette préface, reprenant au mot près un compte rendu de l'ouvrage fait par le philosophe dans Les Temps modernes, n $^{\circ} 137-138$, juillet-août 1957, ne figurera qu'à partir de la réédition Pauvert, postérieure aux indépendances.

4. C'est à quoi nous nous sommes attelés, en collaboration avec l'auteur, dans le cadre du projet Manuscrit Francophone Méditerranée (ITEM CNRS-IRIEC Montpellier III).

5. Albert Memmi, postface inédite à l'édition originale.

6. S'il n'est pas rare qu'un essai soit dès le titre « suivi de... », Ce Portrait du colonisé, précédé d'un Portrait du colonisateur ne me paraît guère d'usage en titraison. Du reste, dans de nombreuses rééditions, seul demeure comme titre Portrait du colonisé. 


\title{
$\underline{\text { Post-face }}$
}

\author{
persuadé \\ eonvaincu \\ 1 - Bien que je sois pé d'une large universalité \\ de leur est
}

de ces deux portraits, que le mécanisme d'émergence seit partout à peu près le même, qu'aucune lecture n'est venue infirmer ces descriptions, je précise qu'elles ont été tracées, pour l'essentiel, d'après des modèles Tunisiens ${ }^{1}$.

2 - J'ai supprimé l'anecdotique et le pittoresque, si tentants dans un tel sujet, mais qui auraient alourdi le dessin sans ajouter à la ressemblance.

De la même, æ j’ai négligé citations, références et sont assez

chiffres, qui m'ont semblé, maintenant, connus largement connus du public. Bien entendu $\mathrm{Il}$ me serait possible

tant

Bien entendu, il me serait possible, d'en faire état.

$$
\text { si besoin, }
$$

\section{Il m'a paru plus import}

3. Il resa à montrer, m'a-t-ilsemble, les traits

3. Il restait à mettre en lumière, m'a-t-il semblé , les traits strueturels des deux protagonistes du $\mathrm{C}^{\mathrm{r}}$ et du $C^{e}$, et teurs liens.

\footnotetext{
${ }^{1}$ J'ai montré dans « Dis-nous d'où tu parles Memmi, ou les embarras du postcolonial » (dans Perspectives européennes des études littéraires francophones, Actes du colloque de Grenoble, à paraître en 2011 aux ELLUG, Grenoble) quels pouvaient être ces modèles, $\pi$ parmi lesquels Jean Amrouche, qui fut le professeur de français de Memmi au lycée Carnot de Tunis et dont on connaît le déchirement durant la guerre d'Algérie.
} 


\section{Post-face}

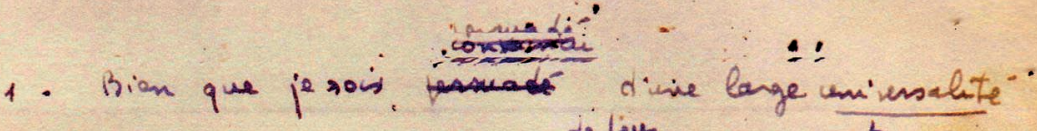

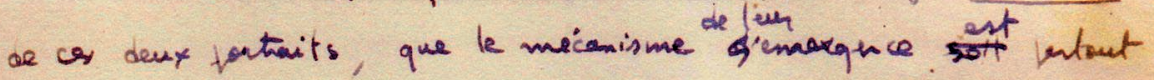
a lou wis lo même, qu'aucune becture n'ast mave ofinfrimes" us

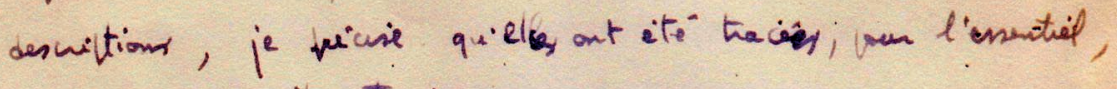
diater der modéter tunisions.

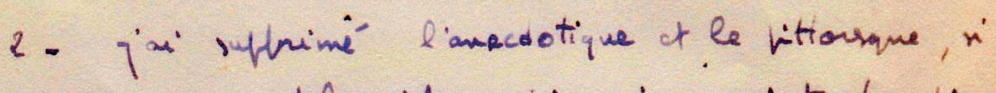
foected tentents dand un tel trjet, mari qui averaint aloundi le dencis sans ajouter $a^{-}$la senenthence.

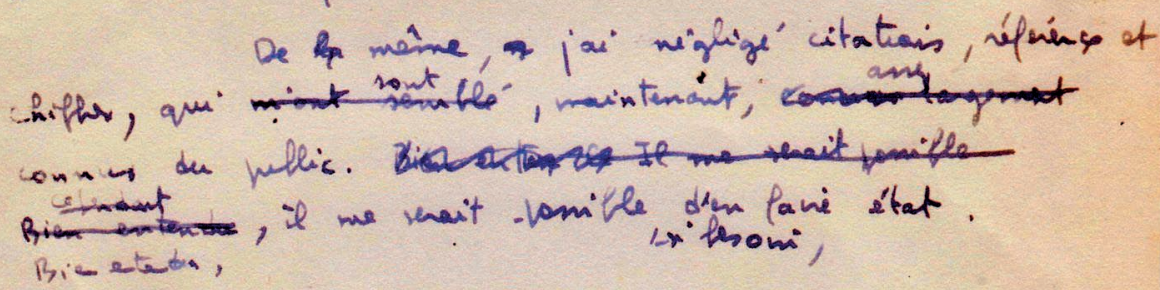
Bionetera,

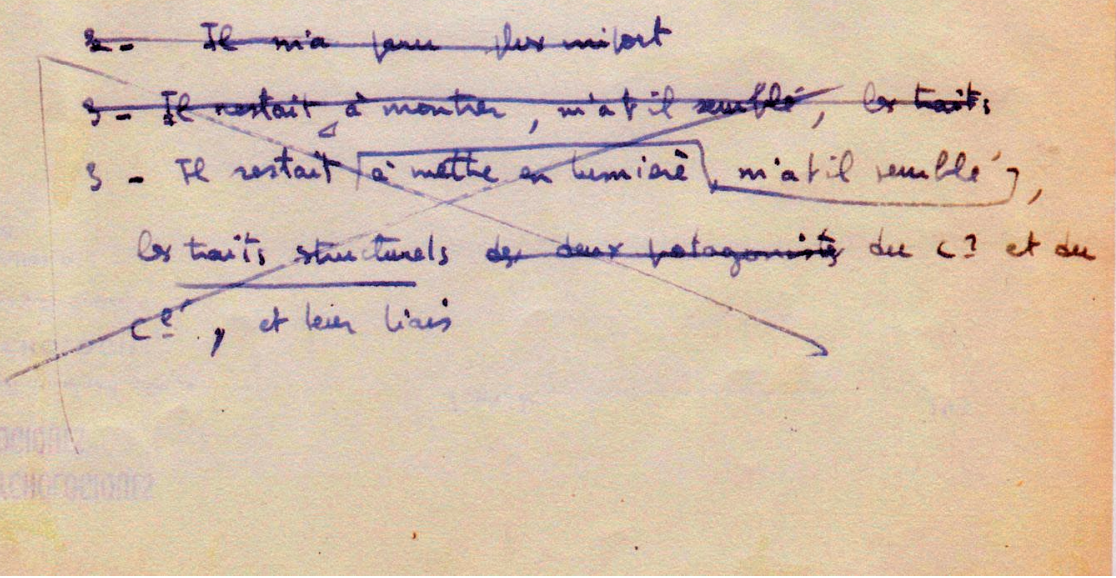

Albert Memmi, « Post-face » au Portrait du colonisé, précédé d’ un Portrait du colonisateur, folios manuscrits 200 à 203, $20,5 \times 20 \mathrm{~cm}$ (coll. privée, (C) Albert Memmi) 
3 - Il restait, me semblait-il, à mettre en lumière, les traits structurels du $\mathrm{c}^{\mathrm{r}}$ et du $\mathrm{c}^{\mathrm{c}}$, et leur liaison fondamentale; la cohérence et la genèse de chaque rôle ; la genèse de l'un par l'autre et la cohérence de la relation coloniale, à partir de la situation coloniale ; En bref, une certaine nécessité du dyptique ${ }^{1}$.

$4^{\circ}$ - C'est pourquoi, je souhaite que ce livre soit lu - ou relu - d'un seul trait, et qu'on en considère le contenu d'une seule vision.

D'une certaine manière, on pourrait y trouver plusieurs portraits : le $c^{r}$ qui se refuse $h$ le $c^{\mathrm{r}}$ qui ou le $\mathrm{c}^{\mathrm{r}}$ de bonne volonté - le $\mathrm{c}^{\mathrm{r}}$ qui s'accepte ou le colonialiste - le $c^{\hat{\varepsilon}}$ qui se refuse ou le candidat à l'assimilation - le $c^{\dot{c}}$ qui se revendique ou la révolte ; sans compter le portrait mythique du $c^{\hat{e}}$. En fait, il n'y a qu'une seule dynamique, celle de la relation coloniale, qui transforme le colonial en $\mathrm{c}^{\mathrm{r}}$, ou en $\mathrm{c}^{\mathrm{iste}}$; et fait souhaiter l'assimilation au cé , puis le pousse à la révolte?

C'est pourquoi, avant de mettre en doute tel ou tel trait, je souhaite qu'on en examine examine à quel moment itse sitte, ilse sittu il appartient. Le $\mathrm{c}^{\mathrm{r}}$ et le $\mathrm{c}^{\hat{\varepsilon}}$ sont, les produits en tant que tels, les

\footnotetext{
${ }^{1}$ Cette «nécessité du diptyque » amènera Memmi à l'élaboration de la notion de duo, qui deviendra par la suite essentielle à sa pensée de la dominance puis de la dépendance.

${ }^{2}$ Dynamique qui avait dans un premier temps conduit à un tout autre plan du Portrait du colonisateur.
} 
3- Te nestait, me subleatil, a' methe en lumine, bs.

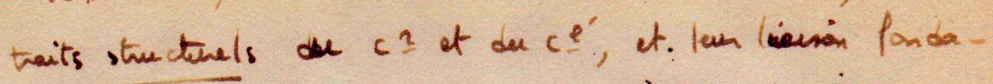
mentale; la cohererce et la gensise de cha quare idle;

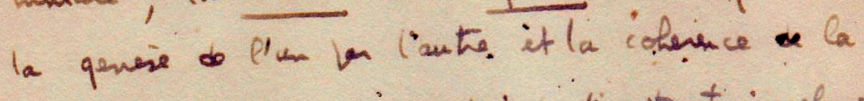

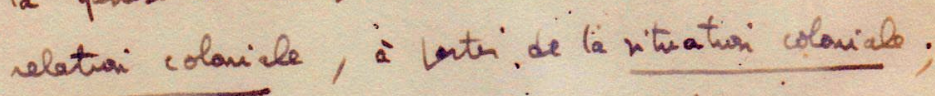
En buel, une certaine récenite an dyitique. .

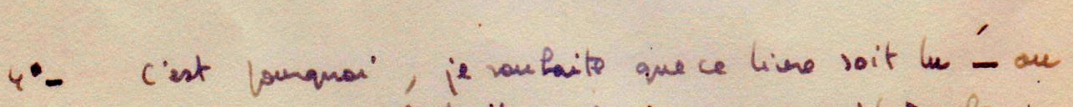
sele - diun sere tait, et quion on considere be contence \& o.une teille virion.

D'une restaine maniers; on loumait y touven flusiount Ditraits: le c qui xenaplese 5 Cespquit on le c? de bonre volonté - le C? qui s'accerte on le colonialiste - be C!'qui se refure ou.

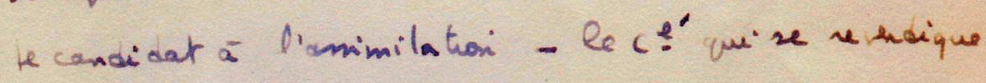
ore la re'velte; Sans comber le hortarit my thique du 1. Er fait, it n'y a qu'unc rule apramique, elle de la velatioi colonicle qui tamplorme le colovial on C?, ou en cirte; ex fait rouhailer innimilatai au cé, jusi le parse à la violte.

Cint burquai a vent de wethe en doute tol on tel tait, ie tou haite quion examine à quel moment il aflertient. le c? et le ce" sont, is are tels, $\&$ 
produits d'une situation historique dont j'ai montré précisément qu'elle est essentiellement instable. Il s'agit, en somme, de deux portraits en mouvement constant.

$5^{\circ} /$ La perspective ici choisie, celle du portrait, rendait superflue une analyse importante de l'aspect économique du fait colonial.

Cependant, si je me suis attaché à décrire le phénomène proprement humain, j’ai assez montré que la relation coloniale naissait d'une situation essentiellement socio-économique ${ }^{1}$. Les notions de privilège et de privilégiéusurpateur $^{2}$ est sont même le point de départ de tout l'itinéraire et une référence toujours sous-jacente.

\footnotetext{
${ }^{1}$ Remarque qui semble répondre à certains reproches faits aux deux portraits parus en revue, qui feraient, selon certains commentateurs, fi de tout contexte socio-économique. On sait en revanche combien Sartre - qui est le premier à formuler ce type de réserve, dans son compte rendu des Temps modernes (op. cit.) - et après lui Fanon, se sont attachés à cet aspect de la situation coloniale.

${ }^{2}$ Lequel conduira Memmi à définir la relation coloniale comme une «pyramide de tyranneaux » au sein de laquelle chacun se trouve dominer l'autre, sauf tout en bas, la large base des indigènes qui ne possèdent rien.
} 


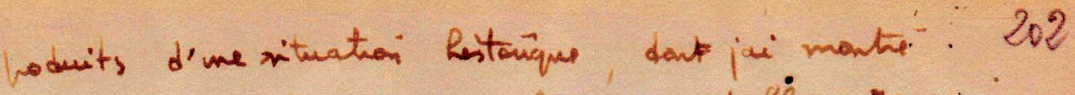
persemist wielle est essentillement wistable. Il sagit en romme, de denx antraits. en movivement constant.

S:/ Ia essective ic choivie, celle du vertait,.

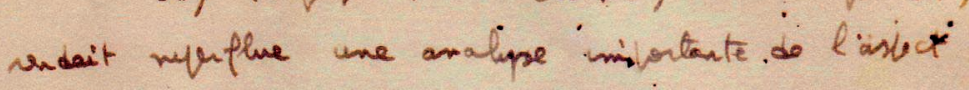
ionomique du fart colorial.

$$
\text { celvibant, ir io we niv attache' is as ".... }
$$

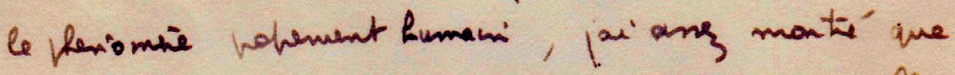
la elation colariale naeriait ine rituatai enstiellement sociozecononique. laprotions de wivileje et de Nvitequé -

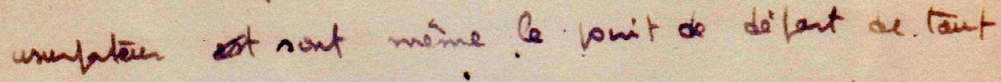

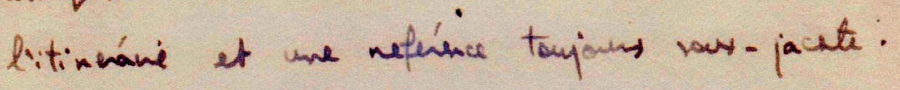


est ensuite divisé en trois chapitres, selon une architecture très scolaire. Celui du « Portrait du colonisé » est établi très tôt dans sa forme définitive; alors que le « Portrait du colonisateur »- avant d'évoluer en parallèle rigoureux avec celui-ci - est plus variable et d'abord fondé sur une variation sur les termes colonial/colonisateur/colonialiste.

Toujours inquiet de la cohérence de son propos, de la logique d'une démarche partant d'une expérience strictement personnelle, l'auteur rédige alors une postface qui ne sera pas retenue par les éditeurs successifs, dans laquelle il justifie « la perspective choisie, celle du portrait », ainsi que ce qu'il appelle «la nécessité du diptyque ». Memmi semble avoir toujours apprécié ce type de discours d'escorte manière à ses yeux de dialoguer avec différentes générations de lecteurs et de tenir compte de l'évolution du monde et des idées. Une analyse en diachronie des différentes préfaces des Portraits s'avérerait du reste très instructive - qu'elles soient autographes, comme celle de la réédition de 1966 (constamment reprise depuis) ou celles de plusieurs rééditions ou traductions de l'essai à l'étranger, ou allographes, comme celles de Jean-Paul Sartre ou, beaucoup plus tard, du président Bouteflika à une réédition algérienne?

Mais c'est à une postface autographe inédite que nous avons choisi de consacrer cette étude ${ }^{8}$. Ce texte de trois pages, qui figure parmi les manuscrits (fos 200 à 202), n'apparaît plus dans les tapuscrits et les épreuves de l'essai. Il est organisé en cinq remarques d'ordre méthodologique, qui traduisent un double souci de l'auteur :

- montrer qu'à partir d'un cas personnel (Memmi a toujours soutenu que sa démarche, embrassant œuvres de fiction et ouvrages de réflexion, le portrait d'une expérience vécue de la domination à la théorisation des mécanismes de celle-ci ${ }^{9}$ ) et d'une situation régionale, celle du Maghreb des années cinquante porté vers les indépendances, on pouvait déboucher, en excluant toute forme d'anecdotique et de pittoresque, sur une « large universalité de ces deux portraits ». En conclusion, l'auteur, s'appuyant cette fois sur sa condition personnelle, définit clairement comme « point de départ de tout [son] itinéraire » et comme « une référence toujours sous-jacente » le rôle d'entre-deux qui ressortit à sa condition ambivalente de juif arabe qui le fait tout à la fois percevoir comme colonisant par les indigènes et comme indigène par le colonisateur 10 ;

- légitimer le genre retenu, celui du portrait (on notera l'importance d'un lexique emprunté au champ pictural : tracé, modèles, pittoresque, dessin, traits...), puis la réunion des deux essais en un seul diptyque cohérent : partant d'un autoportrait, cet essai brosse en fin de compte deux portraits se faisant écho dans un contexte historique et sociologique «en mouvement constant».

Entre les approches politique (Césaire), psychanalytique (Fanon) ou socio-économique (Sartre) de la relation coloniale, Memmi inscrit ainsi ses Portraits dans ce que Suzanne Gearhart nomme « une approche psychoculturelle11 » laquelle, en unissant colonisateur et colonisé en un duo indissociable et pourtant culturellement a-normal, en fait toute l'originalité.

7. Portrait du colonisé, Alger, Éditions ANEP, coll « Voix de l'anticolonialisme », 2006.

8. L'auteur remercie chaleureusement Albert Memmi, qui, en trente ans de collaboration, lui a toujours permis l'accès à ses archives.

9. Voir le discours de réception d'Albert Memmi à l'Académie des Sciences d'Outremer : «De l'expérience vécue à la théorisation ».

10. Jacques Derrida, « né à Alger dans un environnement dont il sera toujours difficile d'affirmer qu'il était colonisateur ou colonisé », exprime la même ambivalence de sa condition dans "The crisis in the teaching of Philosophy ", dans Who's afraid of Philosophy? Right to Philosophy, I, Stanford, Stanford University Press, 2002, p. 103.

11. «Colonialism, psychoanalysis ans cultural criticism: the problem of interiorization in the work of Albert Memmi », dans John Carlos Rowe, Culture ans the problem of the disciplines, New York, Columbia University Press, 1998.

GuY Dugas, né à Uzès en 1950, est actuellement professeur de Littérature générale et comparée (domaine méditerranéen) à l'université Montpellier III, après avoir enseigné plus de quinze ans dans le monde arabe (Tunisie, Maroc et Yémen). Spécialiste du voyage en Orient $\left(\mathrm{XIX}^{\mathrm{e}}-\mathrm{XX} \mathrm{e}^{\mathrm{e}}\right)$ et des expressions minoritaires dans le monde arabo-musulman, il est l'auteur d'une thèse pionnière sur La Littérature judéomaghrébine d' expression française (L’Harmattan, 1990). On lui doit également une somme anthologique sur les francophonies maghrébines (Maroc, Algérie. Un rêve de fraternité; Algérie. Les romans de la guerre et Tunisie. Rêve de partages) aux éditions Omnibus. Au sein de l'équipe qu'il dirige à l'université Montpellier III (IRIEC-EA 740), il a pris en charge la partie « Manuscrit francophone Méditerranée » du projet Manuscrit francophone et a entrepris à ce titre l'édition génétique des Portraits et autres essais d'Albert Memmi.

Guy Dugas, guydugas2002@yahoo.fr 